\title{
Primate Evolution and the Environment
}

Evolution through natural selection is a function of environmental - in the broadest sense - variability through time. Establishing causal relationships between environmental change and specific evolutionary patterns and processes must therefore be one of the central aims of evolutionary biology research.

The present volume grew out of the 2005 Winter Meeting of the Primate Society of Great Britain, held at the Natural History Museum in London, UK. The aim of the meeting was to focus on the influence that environmental factors have or may have had on shaping primate evolution from its origins to modern days. The resulting collection of papers can, of course, neither present a full account of primate evolution nor can it aim to address comprehensively the multitude of environmental variables that are likely to have influenced patterns of evolution in primates. Nevertheless, it does go some way towards demonstrating both the wide range of potential environmental determinants and the great challenges that still lie ahead in terms of achieving an accurate contextualization of evolutionary events in time and space and an understanding of causal relationships between environmental variability and evolutionary change. Two contributions also, importantly, address issues related to the influence that humanity is now having in shaping its own environments as well as those of its fellow primates.

I would like to thank first and foremost all the contributors to this volume for their input and enthusiasm. I would also like to extend my gratitude to all the reviewers for their constructive input into the final publications and to Caroline Harcourt for her expert guidance in bringing this project to fruition. A great number of people contributed to running the meeting on the day, amongst whom I would particularly like to thank Georgina Ash, Silvia Bello, Emmy Bocaege, Gabrielle Delbarre and William Parr. I am particularly grateful for the fact that the Natural History Museum chose to support the meeting by making their conference facilities available at no cost. And last, but certainly not least, I would like to single out the generous sponsorship from Young's brewery in South London, which ensured that speakers and delegates alike were left with positive associations beyond the purely academic content of the day. 